\title{
A Short Report on Single Stage Transcatheter Aortic Valve Replacement and Carotid Stenting
}

\author{
Marco Gennari ${ }^{1}$ Piero Trabattoni ${ }^{1}$ Antonio L. Bartorelli ${ }^{2}$ Marco Agrifoglio ${ }^{3}$ \\ ${ }^{1}$ Centro Cardiologico Monzino, Milano, Lombardia, Italy \\ 2 Department of Biomedical and Clinical Sciences "Luigi Sacco", \\ University of Milan, Milan, Italy \\ Address for correspondence Marco Gennari, MD, Centro \\ Cardiologico Monzino, Via Parea 4, Milan 20138, Italy \\ (e-mail: marcogennari.md@gmail.com).
}

${ }^{3}$ Department of Cardiovascular Sciences and Community Health, University of Milan, Milan, Italy

Thorac Cardiovasc Surg Rep 2017;6:e37-e39.

\author{
Abstract \\ Keywords \\ - transcatheter aortic \\ valve replacement \\ - carotid stent \\ - aortic stenosis \\ - stroke
}

No consensus exists on the timing, safety, and efficacy of treating severe symptomatic aortic and carotid stenosis. In the older population and in the presence of multiple comorbidities that arise during the surgery, a less invasive transcatheter treatment may be the only reasonable option. We discuss this topic by analyzing a case of an 84-yearold man who underwent a combined single-stage transcatheter procedure.

\section{Introduction}

Transcatheter aortic valve replacement (TAVR) has become the preferred treatment option for high-risk surgical patients with severe symptomatic aortic stenosis.

In the PARTNER study, major safety concerns have been raised about the neurological outcome following TAVR. ${ }^{1}$ A large meta-analysis over 1,618 TAVR patients showed a 30-day stroke rate of $6.9 \%{ }^{2}$ Indeed, the population that develops neurological events tends to have a higher mortality rate. The principal cause of the neurological events is considered to be the detachment of aortic debris during catheter manipulation of the calcified valve as well as cerebral embolization during retrograde aortic arch passage of the device.

Furthermore, a significant supra-aortic disease is frequently associated with the valvular lesion. Preoperative assessment must be addressed to minimize the risk of stroke due to supra-aortic trunks disease.

\section{Case}

An 84-year-old Caucasian man was referred for severe symptomatic aortic stenosis. He had a history of hard smoke, coronary artery disease, arterial hypertension, type II diabetes mellitus, chronic renal failure, permanent atrial fibrillation, and a previous right carotid artery stenting. The main symptoms were rest dyspnea and recurrent transient right-side paresthesia and weakness.

The transthoracic echocardiogram showed a low-gradient-low-flow severe aortic stenosis associated with ischemic dilated cardiomyopathy (ejection fraction of $40 \%$ ). An ultrasound scan of the supra-aortic trunks showed patency of the right carotid stent and the presence of severe contralateral carotid stenosis.

The severity of the stenosis (>80\%) according to the North American Symptomatic Carotid Endarterectomy Trial (NASCET) criteria was confirmed by a cervical computed tomography scan; the scan also documented several old bilateral ischemic cerebral lesions.

In consideration of the high surgical risk with a "Society of Thoracic Surgeon" risk score of morbidity or mortality of $32 \%$, we planned a transcatheter approach.

The key question was: Is it safe to perform carotid stenting before TAVR? After Heart Team discussion, we planned a combined transcatheter procedure. Because of the common development of bradycardia and hypotension after carotid stenting and raised risk of asystole in a patient having small aortic orifice area, we performed the aortic valve replacement first.

Heparin was given to achieve a target activating clotting time of 200 seconds. By means of a 14-Fr sheath we implanted via a transfemoral access a 23-mm Edwards SAPIEN received

August 17, 2017

accepted after revision

October 2, 2017
DOI https://doi.org/

10.1055/s-0037-1608887. ISSN 2194-7635. (c) 2017 Georg Thieme Verlag KG
Stuttgart · New York

License terms

(1) $\Theta \circledast$ 


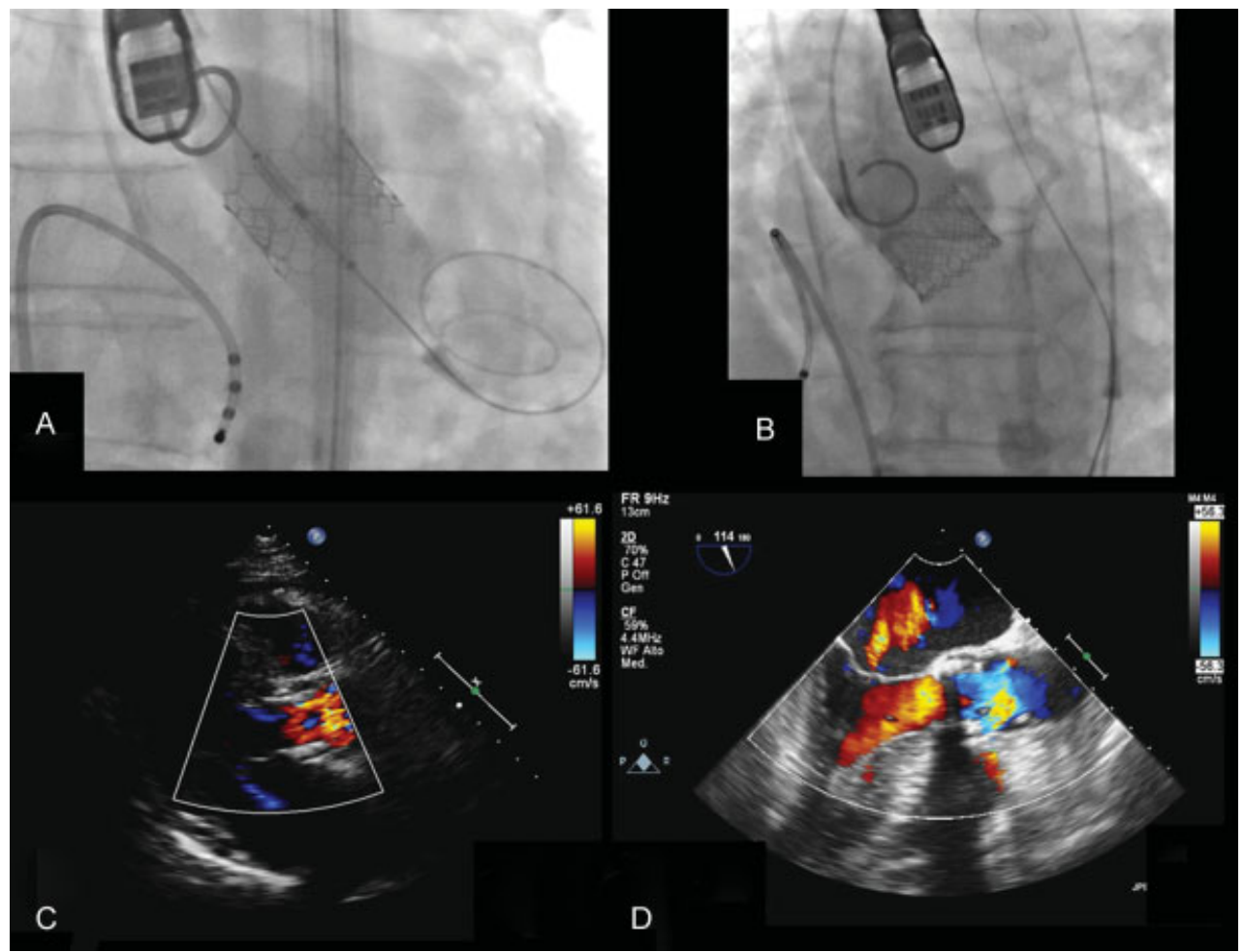

Fig. 1 (A and B) Fluoroscopic image of the delivery of the bioprosthesis. ( $C$ and $D$ ) Intraoperative transesophageal images demonstrating the good result of the implantation.

S3 bioprosthesis (Edwards Lifesciences; Irvine, California United States; - Fig. 1).

After hemodynamic normalization, we implanted a $7 \times 40 \mathrm{~mm}$ Carotid WALLSTENT (Boston Scientific; Massachussets, United States) in the left carotid artery (-Fig. 2). The brain was protected by a 3.2-Fr FilterWire EZ (Boston Scientific) device. The postoperative recovery was uneventful. He is alive and well at 2 years of follow-up.

\section{Discussion}

Currently, there is no consensus on the timing, safety, and efficacy of treating concomitant severe symptomatic aortic and carotid stenosis. Nowadays, in the older population and in those having multiple comorbidities, a transcatheter approach is an attractive option.

Owing to the poor prognosis of these untreated conditions, any effort must be achieved to plan the best treatment in the setting of the multidisciplinary team.

Some authors claim to treat the carotid lesion first, generally 1 month before the TAVR. ${ }^{3}$ The common thought is that the embolic risk is predominantly due to the intrinsic preexisting carotid disease rather than the aortic manipulation.

By contrast, it is known that stroke rate in asymptomatic patients with a carotid stenosis of more than $80 \%$ range from 3.5 to $5 \%{ }^{4}$
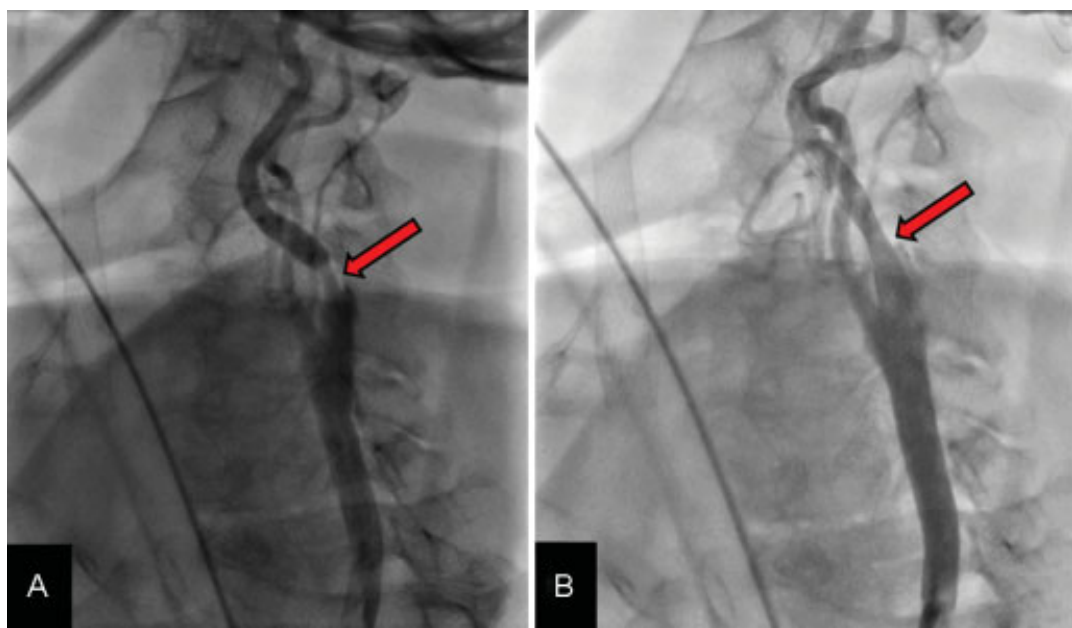

Fig. 2 Angiographic images of the left carotid axis before (A, yellow arrow) and after (B, red arrow) the stenting. 
In these situations, the clinical dilemma is that if we address the valve first, most of the neurological risks still exist due to the embolization of debris from the aortic arch as well as from the valve and from the diseased carotid artery, especially without protection device. On the other hand, the risk of hemodynamic depression during ballooning and deployment of the valve with an impaired cerebral perfusion on the brain side of the severe stenosis may further increase the procedural risk.

We decided to perform a single combined transcatheter procedure to simultaneously treat both conditions in a unique intervention. We performed first the TAVR because we assumed the patient to be at a high risk of hemodynamic instability. And we decided to simultaneously treat both pathological conditions to manage the symptomatic situations in a one-stage fashion.

We still need a broader long-term study on this cohort of patients to effectively evaluate the feasibility and security of this single-stage procedure.

\section{Conflict of Interest}

None.

\section{Funding}

None.

\section{References}

1 Beohar N, Kirtane AJ, Blackstone E, et al. Trends in complications and outcomes of patients undergoing transfemoral transcatheter aortic valve replacement: experience from the partner continued access registry. JACC Cardiovasc Interv 2016;9(04): 355-363

2 Kondur A, Briasoulis A, Palla M, et al. Meta-Analysis of Transcatheter Aortic Valve Replacement Versus Surgical Aortic Valve Replacement in Patients With Severe Aortic Valve Stenosis. Am J Cardiol 2016;117 (02):252-257

3 Ravindra VM, Mazur MD, Kumpati GS, et al. Carotid Artery Stenosis in the Setting of Transcatheter Aortic Valve Replacement: Clinical and Technical Considerations of Carotid Stenting. World Neurosurg 2016;86:194-198

4 Thourani VH, Gunter RL, Neravetla S, et al. Use of transaortic, transapical, and transcarotid transcatheter aortic valve replacement in inoperable patients. Ann Thorac Surg 2013;96(04): 1349-1357 